\title{
A dynamic semantics of single-wh and multiple-wh questions*
}

\author{
Jakub Dotlačil \\ Utrecht University
}

\author{
Floris Roelofsen \\ University of Amsterdam
}

\begin{abstract}
We develop a uniform analysis of single-wh and multiple-wh questions couched in dynamic inquisitive semantics. The analysis captures the effects of number marking on which-phrases, and derives both mention-some and mention-all readings as well as an often neglected partial mentionsome reading in multiple-wh questions.
\end{abstract}

Keywords: question semantics, multiple-wh questions, mention-some, dynamic inquisitive semantics.

\section{Introduction}

The goal of this paper is to develop an analysis of single-wh and multiple-wh questions in English satisfying the following desiderata:

a) Uniformity. The interpretation of single-wh and multiple-wh questions is derived uniformly. The same mechanisms are operative in both types of questions.

b) Mention-some vs mention-all. Mention-some and mention-all readings are derived in a principled way, including 'partial mention-some readings' in multiple-wh questions (mention-all w.r.t. one of the wh-phrases but mention-some w.r.t. the other wh-phrase).

c) Number marking. The effects of number marking on wh-phrases are captured. In particular, singular which-phrases induce a uniqueness requirement in single-wh questions but do not block pair-list readings in multiple-wh questions.

To our knowledge, no existing account fully satisfies these desiderata. While we cannot do justice to the rich literature on the topic, let us briefly mention a number of prominent proposals.

Groenendijk \& Stokhof (1984) provide a uniform account of single-wh and multiple-wh questions, but their proposal does not successfully capture mention-some readings and the effects of number marking.

Dayal (1996) pays due attention to the effects of number marking, but her account does not successfully capture mention-some readings and is not uniform, in the sense that it involves different question operators for single-wh and multiple-wh questions, respectively. Moreover, as pointed out by Xiang $(2016,2020 \mathrm{~b})$ and discussed here below, the so-called domain exhaustivity presupposition that Dayal's account predicts for multiple-wh questions is problematic.

Fox (2012) provides a uniform account of single-wh and multiple-wh questions which captures the fact that singular which-phrases give rise to uniqueness presuppositions in single but not in

* We are grateful to Lucas Champollion, Ivano Ciardelli, Alexandre Cremers, Veneeta Dayal, Danny Fox, Yimei Xiang, and the SALT reviewers and audience for helpful feedback and discussion. We also gratefully acknowledge financial support of the Netherlands Organization for Scientific Research (NWO). 
multiple-wh questions. However, as discussed by Xiang (2016, 2020b), Fox's account, just like Dayal's, derives the problematic domain exhaustivity presupposition for multiple-wh questions. Moreover, while Fox (2013) derives mention-some readings for single-wh questions, it is not clear yet whether his approach could also capture partial mention-some readings in multiple-wh questions, which we will show do exist (see also Xiang 2016). ${ }^{1}$

Finally, Xiang $(2016,2020 b)$ captures mention-some readings and effects of number marking, but her account is not uniform, in the sense that it assumes special LF ingredients for multiple-wh questions which are not present in single-wh questions (see Appendix B for some more details).

The account we propose is crucially dynamic, combining and extending insights from the firstorder dynamic inquisitive semantics (Dotlačil \& Roelofsen 2019) ${ }^{2}$ and the treatment of indefinites and number marking in dynamic semantics (e.g., Brasoveanu 2008).

\section{Informal proposal}

In this section we first present the proposal informally. Some details are suppressed here, so as to provide easy access to the main ideas. The account rests on five key ideas, which are presented in Sections 2.1-2.5, respectively. A formal implementation will be provided in Section 3.

\subsection{Wh-phrases and the witness request operator}

In dynamic semantics a sentence like (1a) gives rise to two consecutive updates of the context. First a discourse referent $u$ is introduced by the indefinite, then the context is updated with the information that $u$ smiled. This sequence of updates is represented in (1b); the exact semantics of this representation will be given in Section 3.

a. Someone smiled.

Now consider the wh-question in (2a). b. $\quad[u] ; \operatorname{smiled}\{u\}$

b. $\quad[u] ; \operatorname{smiled}\{u\} ; ? u$

We analyze wh-phrases just like indefinites: as introducing a discourse referent. But we propose that wh-questions involve an additional update, ? $u$, raising an issue whose resolution requires identifying one or more individuals that have the properties ascribed to $u$-in short, a witness set for $u$. Thus, $(2 \mathrm{a})$ is analyzed as in (2b). We refer to ? $u$ as the witness request operator.

\subsection{Mention-all readings from maximization of witness sets}

As is well-known, when indefinites serve as antecedents for donkey anaphora, two readings can arise, strong and weak. In (3a) the strong reading is most salient, while in (3b) the weak reading is.

1 Fox (p.c.) tells us that, while he was developing the material in Fox 2013 he also noted that mention-some readings are possible in multiple-wh questions, based on examples similar to the ones we will give below. He also had some initial ideas for how to derive such readings, but did not get to develop those ideas at the time.

2 Dynamic inquisitive semantics in turn builds on static inquisitive semantics (Ciardelli, Groenendijk \& Roelofsen 2018) and earlier work on dynamic semantics (in particular that of Groenendijk, Stokhof \& Veltman 1996). For other recent work on questions in dynamic semantics, in the same spirit as ours but focusing on different empirical phenomena, see Li 2019 and Zhao 2019. 
a. If Bill eats an ${ }^{u}$ apple he peels it ${ }_{u}$ first.

b. If Bill has $\mathrm{a}^{u}$ dime he throws it $_{u}$ in the parking meter.

Brasoveanu (2008) argues that the strong reading is derived by a witness maximization operator, $\max \{u\}$, in the interpretation of the indefinite. Intuitively, in (3a) $\max \{u\}$ ensures that all possible values of $u$ (all the apples that Bill eats) are considered in the consequent.

Since we treat wh-phrases just like indefinites, we expect that they can also involve max. Adding $\max \{u\}$ to (2b) results in (4).

$$
[u] ; \operatorname{smiled}\{u\} ; \max \{u\} ; ? u
$$

In order to resolve this question, we have to identify a maximal witness set for $u$. This corresponds to the mention-all reading. On the other hand, the mention-some reading is captured by (2b), without $\max \{u\}$. So, our dynamic semantics of wh-questions makes it possible to connect the mention-some/mention-all ambiguity in wh-questions to the weak/strong ambiguity in donkey anaphora. In other words, the ambiguity between mention-some and mention-all readings is derived in a principled way, relying on a mechanism that is independently needed elsewhere in the grammar. ${ }^{3}$

We will assume that which-phrases, unlike who/where/what, always involve max, so in principle they block mention-some readings. We say 'in principle' because our account correctly predicts that interaction with possibility modals can give rise to mention-some readings even in the presence of max. For reasons of space, however, we cannot discuss this part of the account in detail here.

\subsection{Scaling up to multiple-wh questions}

The account sketched so far is about single-wh questions but can be generalized in a natural way to deal with multiple-wh questions as well. We propose that in a multiple-wh question, each wh-phrase introduces a discourse referent (dref for short), and that the witness request operator asks for a functional witness in this case, i.e., a function mapping the possible values for one dref to the possible values of another. For instance, the multiple-wh question in (5a) is analyzed as in (5b), where $? u_{1} u_{2}$ is a functional witness request operator.

$$
\text { a. Who ate what? }
$$

b. $\left[u_{1}\right] ;\left[u_{2}\right] ;$ ate $\left\{u_{1}, u_{2}\right\} ; ? u_{1} u_{2}$

Informally, ? $u_{1} u_{2}$ raises an issue whose resolution requires determining, for every possible witness set of $u_{1}$, the corresponding witness set of $u_{2}$. Or, in other words, a suitable function from the witness sets of $u_{1}$ to those for $u_{2}$. We will see in Section 3 that both ?u and ? $u_{1} u_{2}$ can be seen as special instances of a general $n$-ary witness request operator, where $n=1$ or $n=2$, respectively. This means that the account is uniform, satisfying desideratum (a).

3 Champollion, Bumford \& Henderson (2019) have recently proposed an alternative strategy to derive strong and weak readings in donkey anaphora, which does not make use of maximization. We leave open here whether this strategy may also be applied in the domain of questions to derive mention-some and mention-all readings. 


\subsection{Partial mention-some readings in multiple-wh questions}

It is typically assumed in the literature that multiple-wh questions only have a mention-all pair-list reading. ${ }^{4}$ For instance, to resolve the question in (6), one must specify, for each party guest, everything that they ate (rather than just something that they ate).

Who ate what at the party last night?

We observe, however, that multiple-wh questions, at least in some cases, also allow for a partial mention-some reading. Suppose Mary has a large herb garden. The herbs that Mary has in her garden each grow in several places. Susan has a list of five herbs that she needs to collect for a certain recipe and asks Mary the following question:

Which of these herbs grows where?

On the most natural reading of this question, Mary can resolve it by mentioning just one location for each herb. She does not have to exhaustively specify all locations for every herb. Our account can straightforwardly derive this reading. Just like in single-wh questions, mention-all readings arise through maximization, and mention-some readings arise in the absence of maximization.

Note however that, while (7) is mention-some w.r.t. locations, it is mention-all w.r.t. herbs. That is, the question requests information about all herbs on the list: it does not suffice to specify just for one herb where it grows. ${ }^{5}$ In English, this appears to be the case in general: multiple-wh questions always require what we will call domain maximality - their resolution must map as many potential witnesses of the 'domain dref' as possible to corresponding witnesses of the 'range dref'. 6

It is important to note that domain maximality as we understand it is different from domain exhaustivity in the sense of Dayal 1996. Domain exhaustivity, adapted to our setting, would require that every potential witness of the 'domain dref' be matched with a corresponding witnesses of

4 It is sometimes argued that multiple-wh questions can have a so-called 'single-pair reading' which is separate from their 'pair-list' reading. In our view, cases in which multiple-wh questions have been reported to have a 'single-pair' reading are just cases where the (most likely) context is one in which only one pair of objects stands in the relation that the question inquires about (see also Dayal 2016: p.95). If this is correct, an account of multiple-wh questions does not need to derive single-pair readings separately from pair-list readings. In any case, we will concentrate here on pair-list readings. If it turns out that there is a need to derive 'single-pair' readings separately, our account will have to be refined.

5 One might think that this is a consequence of the fact that the first wh-phrase which of these herbs is a which-phrase whose domain of quantification is clearly given in the context (it is so-called 'D-linked'). Such wh-phrases are known to favour a mention-all reading in single-wh questions as well. However, the same effect arises with non-D-linked wh-phrases which favor a mention-some reading in single-wh questions. For instance, while (i) has a very salient mention-some reading, (ii) only has a reading that is mention-some w.r.t. one of the wh-phrases, not w.r.t. both.

(i) What is a typical French dish?

(ii) What is a typical dish where?

6 Lucas Champollion (p.c.) has noted that this is not the case in German. For instance, the multiple-wh question in (i) allows for an interpretation which is mention-some w.r.t. both wh-phrases.

(i) Wo kann ich wann Wolfsbarsche fangen?

Where can I when sea bass catch

'Where can I catch sea bass? And when?'

Champollion suggests that this difference between English and German may be connected to the difference between the two languages w.r.t. superiority effects. We leave an exploration of this hypothesis for future work. 
the 'range dref'. Xiang (2019) shows that this requirement is too strong, based on the following scenario. Suppose that 100 candidates have applied for 3 jobs. Mary knows the outcome of the search procedure, but Bill doesn't. Then it is natural for Bill to ask:

Which candidate got which job?

Xiang observes that resolving this question does not require specifying for every candidate which job they got, something that would be impossible to do because there were fewer jobs than candidates. However, an additional observation to make about this example is that a resolution of the question must still specify for as many candidates as possible (in this case three) which jobs they got. This requirement is what we call domain maximality.

In our formal account in Section 3, domain maximality will be captured by an operator that is always present in the left periphery of an interrogative clause. In particular, it will be derived no matter whether a max operator applies to the domain dref or not. So our account will derive two readings for multiple-wh questions: one that is mention-all with respect to all wh-phrases, and one that is mention-all with respect to the 'domain wh-phrase(s)' but mention-some with respect to the 'range wh-phrase'. For this to be possible, it is crucial that on our account mention-some is not a property connected to a question as a whole but rather to individual wh-phrases. In this respect our account differs from many others (e.g., Beck \& Rullmann 1999, Chapter 2 of George 2011, Theiler 2014, Champollion, Ciardelli \& Roelofsen 2015), which take mention-some to be a property connected to questions as a whole. On such accounts, it is not possible to derive partial mention-some readings of multiple-wh questions (at least not without substantial modifications). To our knowledge, the only previous account that derives such readings is that of Xiang 2016: §5.4.3.

\subsection{Effects of number marking}

So far we have outlined an account that satisfies desiderata (a) and (b). We now turn to desideratum (c), which concerns the role of number marking. Several effects need to be captured but the main puzzle is that singular which-phrases induce a uniqueness presupposition in single-wh questions but allow for multiple witness-pairs in multiple-wh questions (e.g., Dayal 1996). ${ }^{7}$

$$
\begin{aligned}
& \text { Which girl danced with Robin? } \\
& \sim \text { just one girl danced with Robin }
\end{aligned}
$$

Which girl danced with which boy? $\not \rightarrow$ just one girl and just one boy danced

We will assume, following Brasoveanu (2008) and much other work on number marking in dynamic semantics, that singular number marking, e.g., on which girl, invokes an atomicity requirement. This ensures that all the possible values assigned to the dref introduced by which girl are atomic individuals. Furthermore we will assume, following Roelofsen (2015), that every interrogative clause involves a presuppositional closure operator, $\dagger$, which requires that it is already known in

7 Interestingly, it has recently been observed that in single-wh questions with an existential modal operator, the uniqueness requirement normally induced by a singular which-phrase can be obviated (Hirsch \& Schwarz 2019). For instance, it is perfectly natural to ask (i) without assuming that there is a unique letter that can be inserted in fo_ $m$ to make a word.

(i) Which letter can be inserted in fo_ $m$ to make a word?

Our account, when extended with a treatment of modality, could account for this and related facts. However, for reasons of space, we must leave such an extension for another occasion. 
the input context that the actual world lies in one of the alternatives that the question introduces. This presupposition is trivially satisfied whenever the alternatives cover the entire logical space, but not when the alternatives cover only part of the logical space.

A question like (9), repeated in (11a), is then analyzed as in (11b):

$$
\begin{aligned}
& \text { a. Which }{ }^{u} \text { girl danced? } \\
& \text { b. } \dagger([u] ; \operatorname{atom}\{u\} ; \operatorname{girl}\{u\} ; \operatorname{danced}\{u\} ; \max \{u\} ; ? u)
\end{aligned}
$$

As we will see in detail in Section 3, a uniqueness presupposition is derived in this case from the interplay between atom $\{u\}, \max \{u\}, ? u$, and $\dagger$. A particularly important role is played by ?u. This operator removes any possibilities from the context where more than one girl dances.

For a multiple-wh question we get:

$$
\begin{aligned}
& \text { a. Which }{ }^{u_{1}} \text { girl danced with which }{ }^{u_{2}} \text { boy? } \\
& \text { b. } \dagger\left(\begin{array}{c}
{\left[u_{1}\right] ; \operatorname{atom}\left\{u_{1}\right\} ; \operatorname{girl}\left\{u_{1}\right\} ;\left[u_{2}\right] ; \operatorname{atom}\left\{u_{2}\right\} ; \operatorname{boy}\left\{u_{2}\right\} ;} \\
\text { danced_with }\left\{u_{1}, u_{2}\right\} ; \max \left\{u_{1}\right\} ; \max \left\{u_{2}\right\} ; ? u_{1} u_{2}
\end{array}\right)
\end{aligned}
$$

A global uniqueness requirement is not derived here because, instead of ? $u$, the two-place witness request operator ? $u_{1} u_{2}$ is at play in this case. This does not remove possibilities from the input context where more than one girl or more than one boy danced, but only ones where there is no function mapping each girl to the unique boy she danced with. As a result, the account derives a 'relativized' uniqueness presupposition to the effect that no girl danced with more than one boy.

\section{Implementation}

We implement the above ideas in a dynamic type-theoretic framework. We assume four basic semantic types: $e$ for atomic and plural entities (e.g., $a, a \oplus b$ ), $s$ for possible worlds, $t$ for truth values, and $r$ for discourse referents (cf. Muskens 1996; Brasoveanu 2008). We refer to functions from discourse referents to entities as discourse referent assignment functions or (dref) assignments for short. As a convention, we use the variable $g$ for dref assignments. A set of dref assignments can be thought of as a dref assignment matrix, where each assignment function determines one row of the matrix (van den Berg 1996; Brasoveanu 2008). Such sets of dref assignments are represented by capital letters $G, G^{\prime}$ and their type, $\langle r e\rangle t$, is abbreviated as $m$.

As is standard in inquisitive semantics, we take a context to be a downward closed set of information states. Every information state consists of possibilities and each possibility is a pair $\langle w, G\rangle$, where $w$ is a world and $G$ a set of assignments mapping discourse referents to (atomic or plural) entities. For contexts, we use the variable $c$, for information states, we use the variable $s$.

We visualize contexts by means of diagrams such as the one on the right. Each row corresponds to an assignment set $G$, each column to a world $w .{ }^{8}$ Each black dot is a possibility $\langle w, G\rangle$, where $w$ is determined by the column and $G$ by the row. In most examples, we will assume that $G$ consists of just one assignment, e.g., $u / a$ in the top row represents the case in which $G$ consists of a single assignment that assigns $a$ to

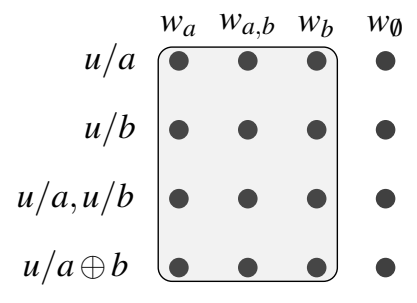

8 We subscript $w$ with letters representing entities. This should be read as 'a world in which the subscripted entity has a property $P^{\prime}$. In the following subsection, $P$ is the property of smiling. In Section 3.3, $P$ corresponds to whatever property is introduced in examples. 
the dref $u$. If there are more assignments in $G$, we separate them by comma (see $u / a, u / b$ in the third row) or, when dealing with several discourse referents, we write the assignments in $G$ underneath each other.

Each shaded area is a maximal information state in the context (also called an alternative). To keep the diagrams simple, we will often assume that the context involves just two atomic entities, $a$ and $b$, and their sum $a \oplus b$.

If a context contains just one alternative, as in the diagram above, then there are no open issues. We say that the context is non-inquisitive in this case. On the other hand, if there are two or more alternatives, or no alternatives at all (which is only possible if there are infinitely many possibilities), the context contains unresolved issues and we say that it is inquisitive.

\subsection{Context updates}

Context update functions are functions from contexts to contexts. For our purposes, it suffices to define the following seven update functions: (i) basic updates conveying that certain drefs have certain properties, e.g., smile $\{u\}$, (ii) updates that introduce a new dref, e.g., $[u]$ (iii) updates that request a (functional) witness, e.g., ? $u$ and $? u_{1} u_{2}$, (iv) updates that maximize the witness set of a dref: $\max \{u\},(\mathrm{v})$ updates that impose an atomicity requirement on the witnesses associated with a dref, e.g., atom $\{u\}$, and finally (vi) updates involving the presuppositional closure operator $\dagger$.

Let us start with a simple predicational update function: smile $\{u\}$ takes an input context $c$ and returns a new context, which is the set of information states $s$ in $c$ such that for every possibility $\langle w, G\rangle$ in $s$ and every assignment $g$ in $G$, it holds that $g(u)$ smiles in $w$. We assume, as is common, that atomic predicates like smile are pluralized (Krifka 1989; Landman 2000; Kratzer 2008). This is signaled by the $*$ preceding the predicate. The definition of $*$ is given in (14) for a relation of an arbitrary arity $n$.

$$
\begin{aligned}
& \text { smile }\{u\}:=\lambda c_{\langle\langle\langle s \times m\rangle t\rangle t\rangle} \lambda s_{\langle\langle s \times m\rangle t\rangle} . s \in c \wedge \forall\langle w, G\rangle \in s . \forall g \in G . * \operatorname{smile}(w)(g(u)) \\
& \text { a. } \quad R \subseteq * R \\
& \text { b. } \quad \text { If }\left\langle\alpha_{1}, \ldots \alpha_{n}\right\rangle \in * R \text { and }\left\langle\alpha_{1}^{\prime}, \ldots \alpha_{n}^{\prime}\right\rangle \in * R, \text { then also }\left\langle\alpha_{1} \oplus \alpha_{1}^{\prime}, \ldots \alpha_{n} \oplus \alpha_{n}^{\prime}\right\rangle \in * R . \\
& \text { c. } \quad \text { Nothing else is in } * R .
\end{aligned}
$$

The predicational update function smile $\{u\}$ can be generalized to the n-ary relational update function $R\left\{u_{1}, \ldots u_{n}\right\}$ in (15):

$$
R\left\{u_{1}, \ldots u_{n}\right\}:=\lambda c_{\langle\langle\langle s \times m\rangle t\rangle t\rangle} \lambda s_{\langle\langle s \times m\rangle t\rangle} . s \in c \wedge \forall\langle w, G\rangle \in s . \forall g \in G . * R(w)\left(g\left(u_{1}\right)\right) \ldots\left(g\left(u_{n}\right)\right)
$$

The effect of (13) is represented graphically in the diagram below. In the diagram, the context on the left is the input context, the one on the right the output context. The update, smile $\{u\}$, eliminates those possibilities in the input context in which at least one entity assigned to $u$ does not smile. 


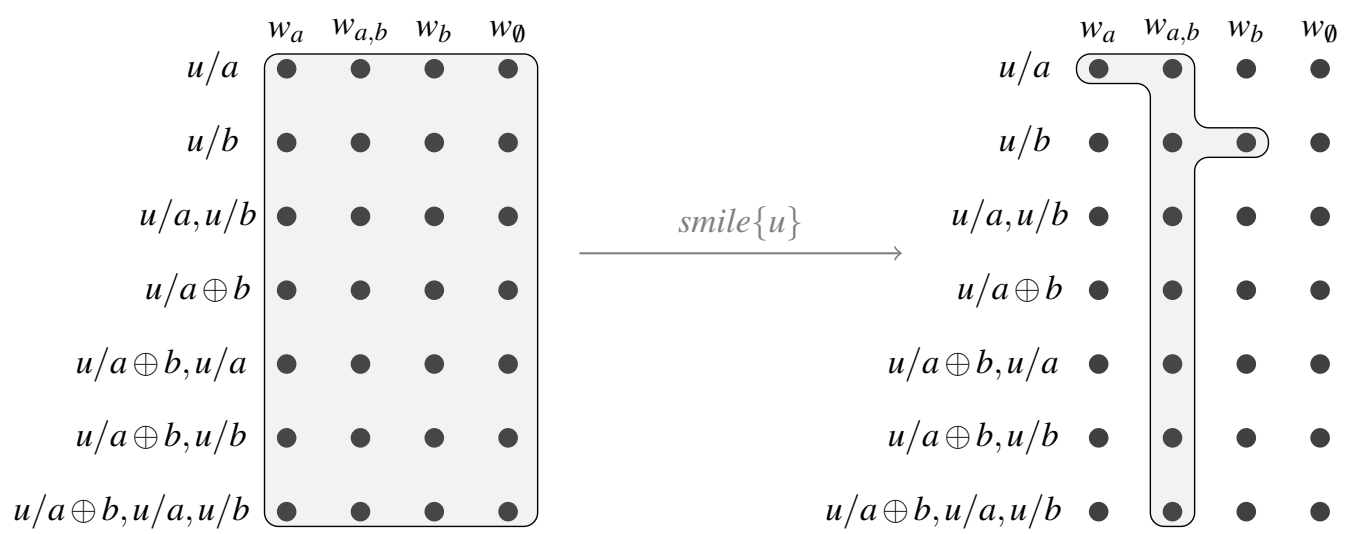

The update function $[u]$ introduces a discourse referent $u$. Intuitively, $[u]$ updates a state in the input context by randomly assigning any possible values to $u$ across the assignments of the possibilities in the state. The full formal definition is given in (16). It is a pointwise generalization of dref introduction in Brasoveanu (2008) to contexts in dynamic inquisitive semantics.

$$
[u]:=\lambda c_{\langle\langle\langle s \times m\rangle t\rangle t\rangle} \lambda s_{\langle\langle s \times m\rangle t\rangle} \cdot \exists s^{\prime} \in c .\left(\begin{array}{l}
\forall\langle w, G\rangle \in s . \exists\left\langle w, G^{\prime}\right\rangle \in s^{\prime} .\left(G^{\prime}[u] G\right) \wedge \\
\forall\left\langle w, G^{\prime}\right\rangle \in s^{\prime} . \exists\langle w, G\rangle \in s .\left(G^{\prime}[u] G\right)
\end{array}\right)
$$

$G^{\prime}[u] G$, used in (16) for dref introduction, is defined in the same way as dref introduction in Brasoveanu (2008). The definition is given in (17), where $g^{\prime}[u] g$ is true when $g^{\prime}$ only differs from $g$ with respect to a, possibly newly introduced, discourse referent $u$ (see Muskens 1996 for details).

$$
G^{\prime}[u] G:=\forall g \in G . \exists g^{\prime} \in G^{\prime} \cdot g^{\prime}[u] g \wedge \forall g^{\prime} \in G^{\prime} . \exists g \in G . g^{\prime}[u] g
$$

The effect of $[u]$ is illustrated in the diagram below. Notice that after the update $u$ can carry any possible value in any column. If the context is further updated with smile $\{u\}$, only those possibilities are kept which are such that the entities assigned to $u$ smile.

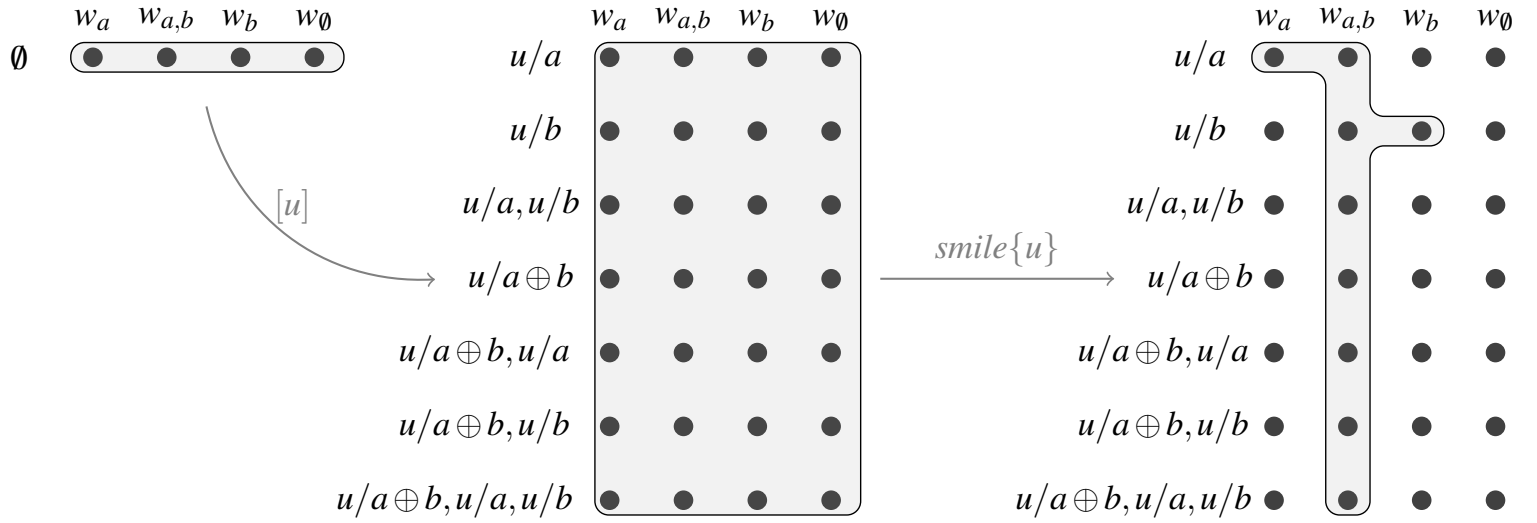

The next update function is the witness request operator ? $u$, defined in (18). $? u$ selects those states $s$ in the input context which contain enough information about the world to ensure the existence of a witness (atomic or plural entity) for $u$.

$$
? u:=\lambda c_{\langle\langle\langle s \times m\rangle t\rangle t\rangle} \lambda s_{\langle\langle s \times m\rangle t\rangle} . s \in c \wedge \exists x_{e} . \forall\langle w, G\rangle \in s . \exists\left\langle w, G^{\prime}\right\rangle \in \bigcup c . \forall g^{\prime} \in G^{\prime} . g^{\prime}(u)=x
$$

A graphical illustration of the workings of ? $u$ is given in the diagram below. Note that, in this example, ? $u$ does not eliminate any possibilities. The output context contains the same information 
as the input context, but the output context has turned inquisitive: two alternatives have been introduced. That is, ? $u$ raises an issue, which could be paraphrased as 'Which individual has the properties ascribed to $u$ ? Is $a$ such an individual (the alternative extending to the left), or is $b$ such an individual (the alternative extending to the right)?' This issue-raising update effect of ? $u$ will play a crucial role in our account of wh-questions.

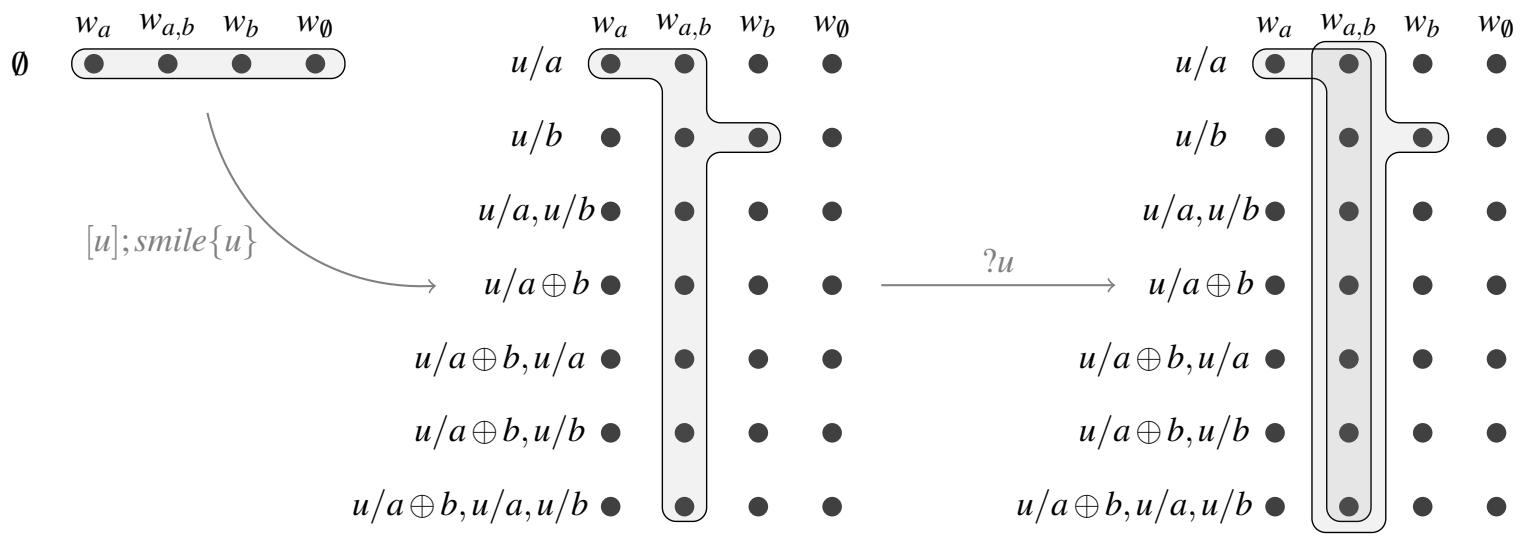

For multiple-wh questions, we need a more general witness request operator, $? u_{1} \ldots u_{n}$, whose update effect is defined in (19). This operator selects states in the input context which contain enough information about the world to ensure the existence of a witness function, mapping values of $u_{1} \ldots u_{n-1}$ to suitable values of $u_{n}$. Crucially, as readers can check, ?u is just a specific instance of $? u_{1} \ldots u_{n}$, where $n=1$. Because of this, single-wh and multiple-wh questions can be dealt with in a uniform way on our account, as we will see in more detail in Section 3.3.

$$
\begin{array}{r}
? u_{1} \ldots u_{n-1} u_{n}:=\lambda c_{\langle\langle\langle s \times m\rangle t\rangle t\rangle} \lambda s_{\langle\langle s \times m\rangle t\rangle} . s \in c \wedge \exists f_{\left(e^{n-1}, e\right)} . \forall\langle w, G\rangle \in s . \exists\left\langle w, G^{\prime}\right\rangle \in \bigcup c . \\
\forall g^{\prime} \in G^{\prime} .\left(g^{\prime}\left(u_{n}\right)=f\left(g^{\prime}\left(u_{1}\right), \ldots, g^{\prime}\left(u_{n-1}\right)\right)\right.
\end{array}
$$

The next update function is $\max \{u\}$, which restricts the context to states consisting of possibilities in which the 'cumulative value' of $u$ is maximal, compared to other possibilities with the same world parameter. The cumulative value of $u$ in a possibility $\langle w, G\rangle$, notated as $\oplus G(u)$, is the sum of all individuals assigned to $u$ across the assignments in $G$. A graphical illustration is given below the definition.

$$
\begin{aligned}
& \max \{u\}:=\lambda c_{\langle\langle\langle s \times m\rangle t\rangle t\rangle} \lambda s_{\langle\langle s \times m\rangle t\rangle} . s \in c \wedge \forall\langle w, G\rangle \in s . \forall\left\langle w, G^{\prime}\right\rangle \in \bigcup c . \oplus G^{\prime}(u) \subseteq \oplus G(u)
\end{aligned}
$$

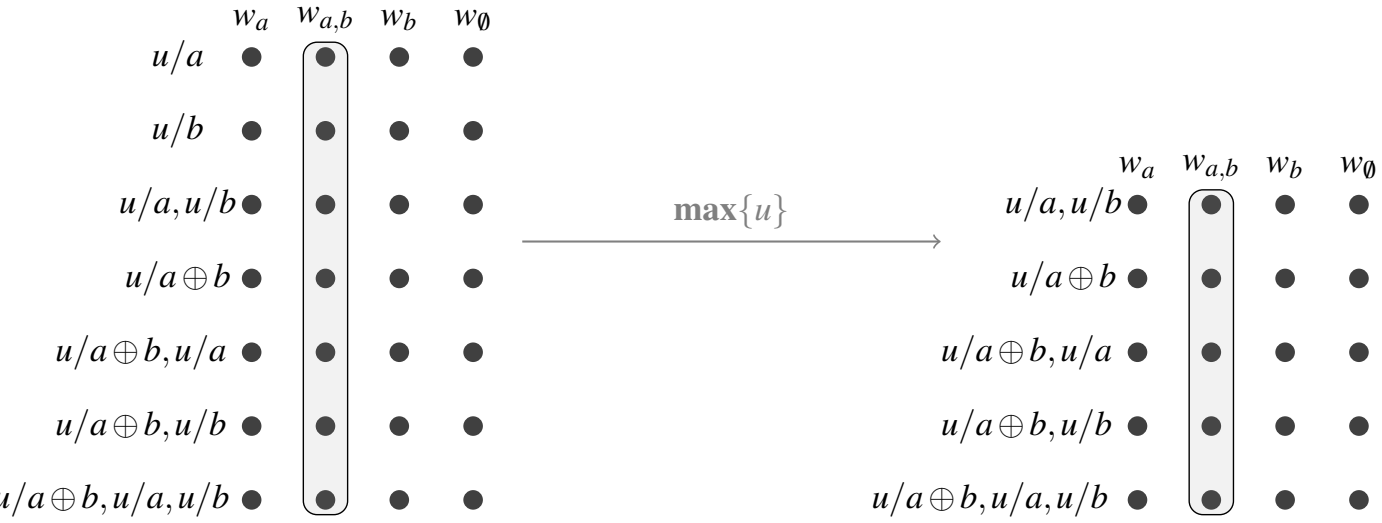


The basic max operator defined in (20) suffices to derive strong readings of donkey anaphora and mention-all readings of single-wh questions. However, for multiple-wh questions we need a somewhat more refined operator, defined in (21). This operator restricts a context $c$ to states consisting of possibilities whose dref assignment functions collectively assign a maximal set of individuals to $u$, independently of the values assigned to other drefs. In (21), $G^{\vec{u} \backslash u_{n} \mapsto \vec{x}}\left(u_{n}\right)$ is the set of values assigned to $u_{n}$ by assignments in $G$ which pointwise assign the values $\vec{x}$ to $\vec{u} \backslash u_{n}$, all drefs except $u_{n}$. If $u_{n}$ is the only dref, then $\max ^{*}\left\{u_{n}\right\}$ just amounts to $\max \left\{u_{n}\right\}$. But if there are other drefs around, the two may come apart.

$$
\begin{array}{r}
\max ^{*}\left\{u_{n}\right\}:=\lambda c_{\langle\langle\langle s \times m\rangle t\rangle t\rangle} \lambda s_{\langle\langle s \times m\rangle t\rangle} . s \in c \wedge \forall\langle w, G\rangle \in s . \forall\left\langle w, G^{\prime}\right\rangle \in \bigcup c . \\
\forall \vec{x} . \oplus G^{\prime \vec{u} \backslash u_{n} \mapsto \vec{x}}\left(u_{n}\right) \subseteq \oplus G^{\vec{u} \backslash u_{n} \mapsto \vec{x}}\left(u_{n}\right)
\end{array}
$$

The workings of $\mathbf{m a x}^{*}$ are illustrated in the diagram below. Notice that there are two discourse referents in this example. $\mathbf{m a x}^{*}$ applies to $u_{2}$. The output context contains only those possibilities in which $u_{2}$ is maximal for any value of $u_{1}$. Concretely, the top possibility in the input context is eliminated because $u_{2}$ is not maximal for any value of $u_{1}$ compared to the two other possibilities.

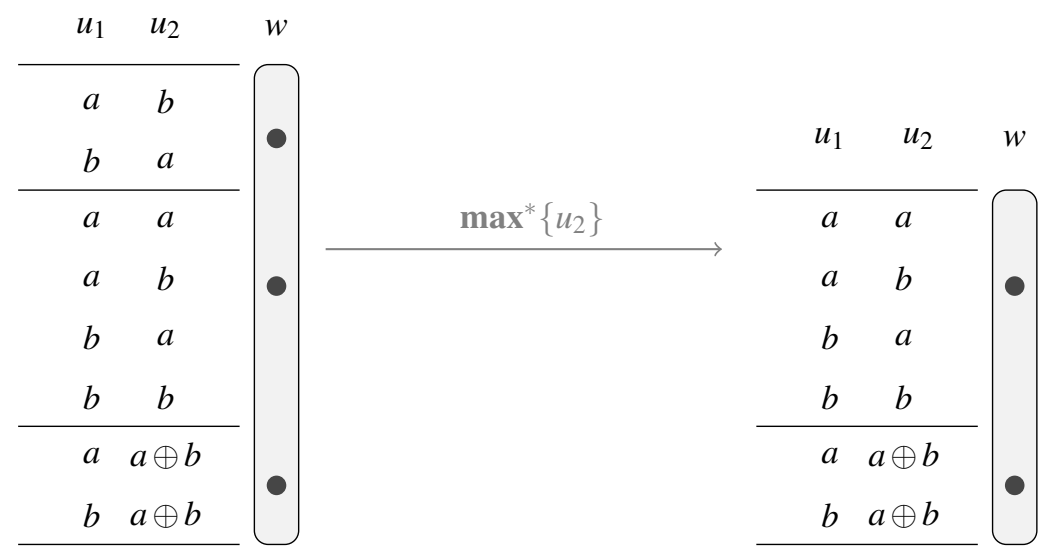

Next, we define an update function requiring atomicity of the individuals assigned to $u$ :

$$
\operatorname{atom}\{u\}:=\lambda c_{\langle\langle\langle s \times m\rangle t\rangle t\rangle} \lambda_{\langle\langle s \times m\rangle t\rangle} . s \in c \wedge \forall\langle w, G\rangle \in s . \forall g \in G . \neg \exists y(y<g(u))
$$

The atomicity requirement atom $\{u\}$ eliminates those possibilities in which the value of $u$ is nonatomic, as illustrated in the following diagram.

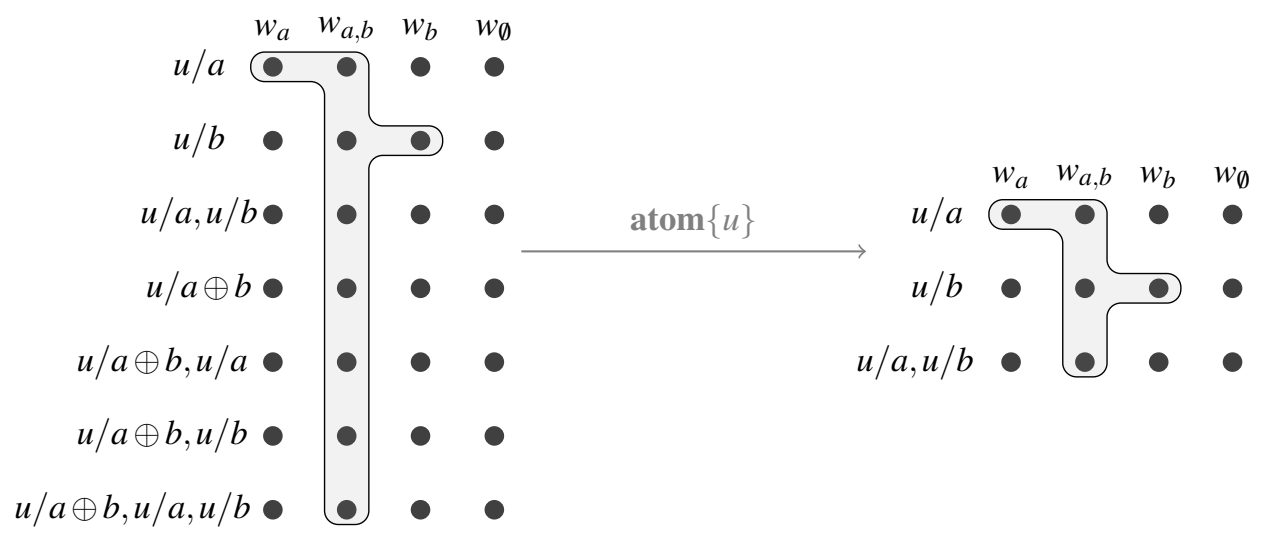


The final operator to be defined is the presuppositional closure operator, $\dagger$. In the definition in (23), $U$ is an arbitrary update function. The notion of subsistence that the definition refers to is specified in Appendix A.

$$
\dagger U:=\lambda c_{\langle\langle\langle s \times m\rangle t\rangle t\rangle} \cdot \begin{cases}U(c) & \text { if } \bigcup c \text { subsists in } \bigcup U(c) \\ \text { undefined } & \text { otherwise }\end{cases}
$$

The dagger operator ensures that all the worlds and dref value combinations considered possible in the input context are still considered possible in the output context, i.e., none of them are removed by the update function $U$. If this is not the case, the update $\dagger U$ is undefined. This means that $\dagger U$ can only raise issues and/or introduce discourse referents, it never adds any other information. The diagram below contains an output context (on the right) and two input contexts, $c_{1}$ and $c_{2}$. Suppose $U$ is an update function which, when applied to either of these input contexts, yields the output context on the right. Then, $\dagger U\left(c_{1}\right)$ is defined but $\dagger U\left(c_{2}\right)$ is undefined, because $w_{a, b}$ is considered possible in $c_{2}$ but not in the output context.
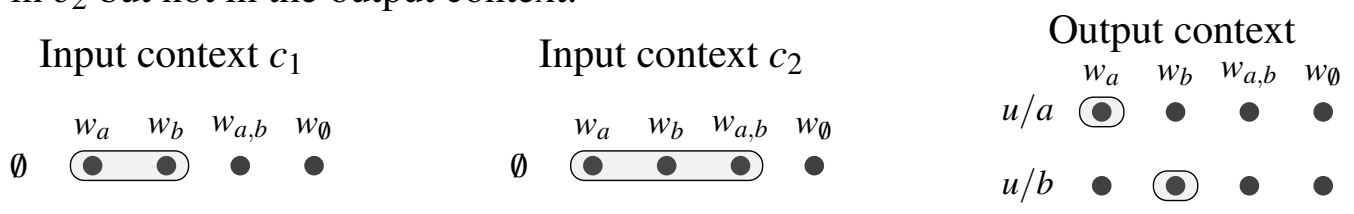

\subsection{Syntax-semantics mapping in wh-questions}

We are almost ready to see how wh-questions are interpreted on our account. First, however, we need to specify some basic assumptions about the syntax-semantics interface. For reasons of space, we only discuss the left periphery of clauses in some detail. For the rest, standard assumptions from compositional dynamic systems (e.g., Muskens 1996) are adopted.

We assume that there are two projections in the left periphery of wh-questions, a Type projection and a Foc projection (cf., Rizzi 1997, 2001):

$$
\text { Left periphery of wh-questions: } \quad[\text { TypeP }[\text { Type }][\text { FocP }[\text { Foc }][T P]]]
$$

The lower head, Foc, introduces a witness request operator over the drefs introduced by wh-phrases that stand in an Agreement relation with Foc. Furthermore, we assume that Foc requires maximality of all associated drefs except the last, $u_{n}$, which, as we will see, ensures that in multiple-wh questions, all associated wh-phrases except for one obtain a mention-all interpretation.

$$
\left[\left[\operatorname{Foc}_{u_{1}, \ldots u_{n}}\right]\right]:=\lambda U_{\mathbf{T}} . U ; \mathbf{m a x}^{*}\left\{u_{1}\right\} ; \ldots ; \mathbf{m a x}^{*}\left\{u_{n-1}\right\} ; ? u_{1} \ldots u_{n}
$$

where $u_{1}, \ldots u_{n}$ are the drefs introduced by wh-phrases that Agree with Foc

The higher head, Type, adds the presuppositional closure operator, $\dagger$.

$$
[[\text { Type }]]=\lambda U_{\mathbf{T}} \cdot \dagger U
$$

Two other assumptions not tied to the left periphery should be mentioned. First, we assume that which-phrases always carry a maximality requirement. Due to this assumption, which-phrases always generate mention-all readings in our analysis here (though this would no longer be the case if modal operators were taken into consideration, see Section 2.2 above). Second, we assume that singular number marking (e.g., on singular which-phrases) induces an atomicity requirement. 
This broad and unconstrained specification of the syntax-semantics interface is sufficient for our purposes here. Further details can be added to provide an account that also covers polar and alternative questions, but we will not pursue these extensions here.

\subsection{Illustrations}

Before illustrating the predictions of the account for single-wh and multiple-wh questions, we first briefly consider a simple declarative sentence with an indefinite referential expression.

$$
\text { Someone smiles. } \leadsto[u] ; \text { smile }\{u\}
$$

Let us assume for this and the following example that there are only two atomic individuals, $a$ and $b$, and we start with a context in which no discourse referent is present yet and it is open who of $a$ and $b$, if anyone, smiled. Then, (27) gives rise to the update displayed below.

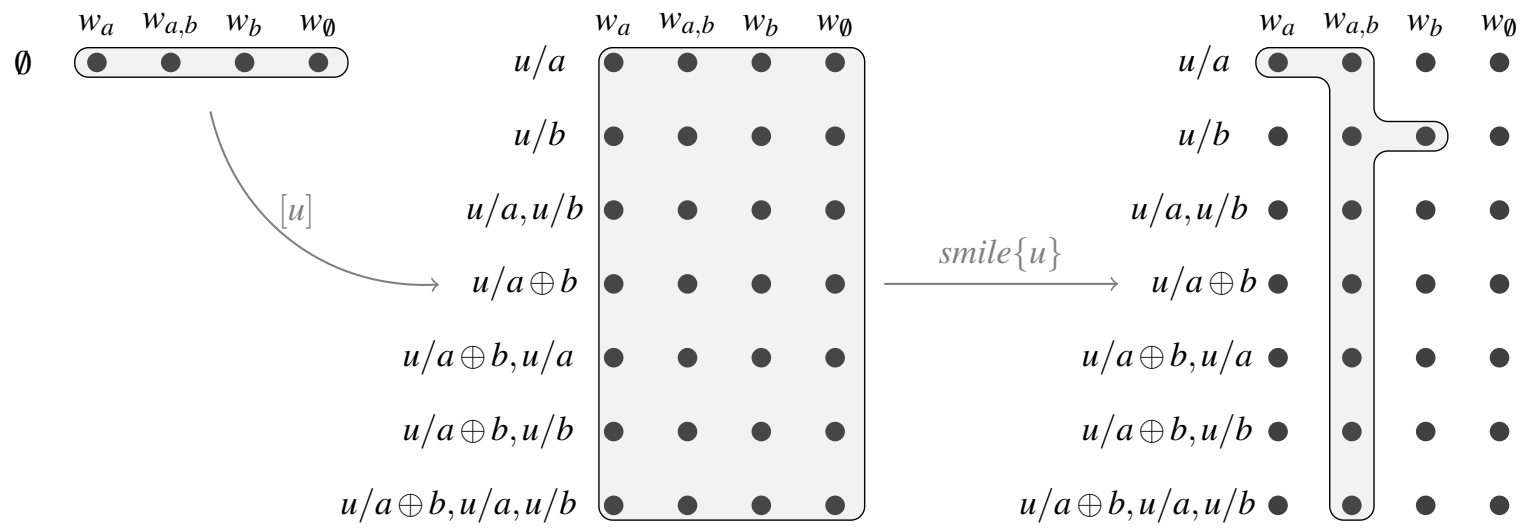

A single-wh question on its weak reading is translated as in (28). The wh-word is interpreted just like an indefinite. The Foc head in the left periphery contributes the witness request operator ? $u$, and the interrogative Type head contributes the $\dagger$ operator.

$$
\text { Who }_{\text {weak }} \text { smiles? } \leadsto \dagger([u] ; \text { smile }\{u\} ; ? u)
$$

Due to ? $u$, the question raises an issue, as seen in the last step in the diagram below. Due to the $\dagger$ operator, the input context for the question must exclude $w_{\emptyset}$, otherwise the update is undefined. Thus, due to $\dagger$, the question presupposes that someone smiles.

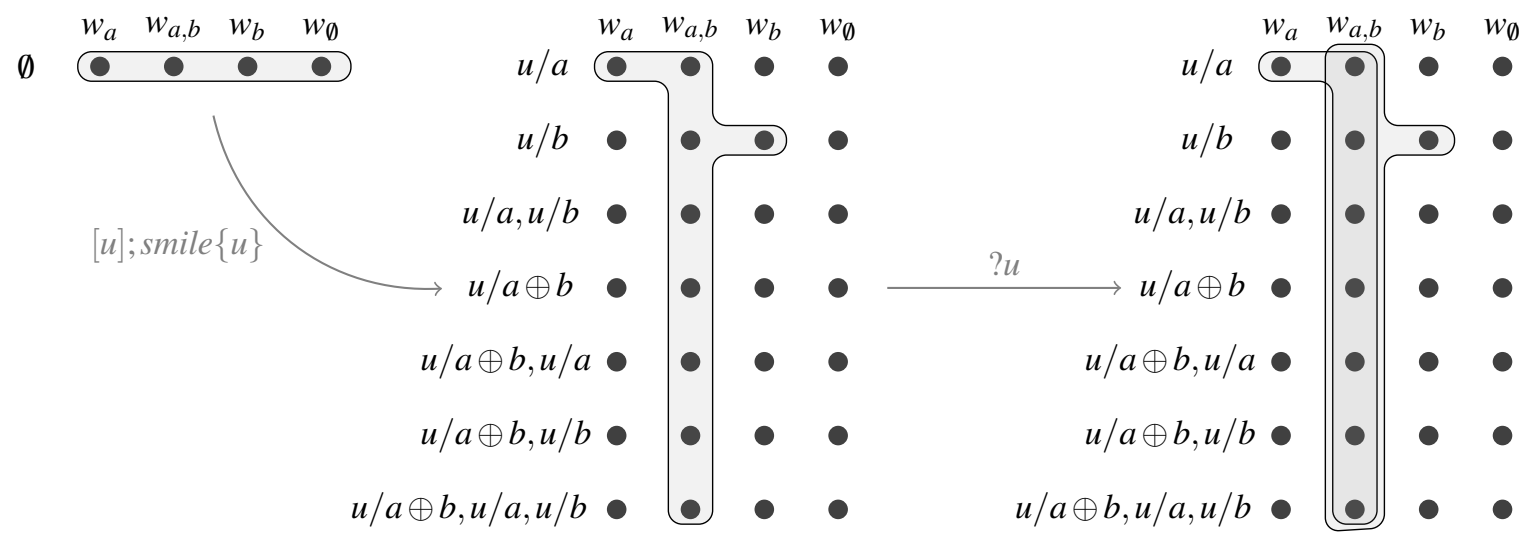

The strong reading of the same question is derived as in (29), where $\max ^{*}\{u\}$ enters the 
stage. $\max ^{*}\{u\}$ removes possibilities whose assignments cumulatively assign a non-maximal plural individual to $u$ compared to other possibilities with the same world-parameter (i.e., in the same column of the diagram). Unlike in the previous case, every information state in the output context now consists of possibilities which cumulatively assign the maximal plural individual to $u$ that smiled according to the worlds in the information state. This generates a mention-all reading. For example, to resolve the question in (29), one has to specify the maximal plurality of individuals that smiled-just mentioning one of them is not sufficient.

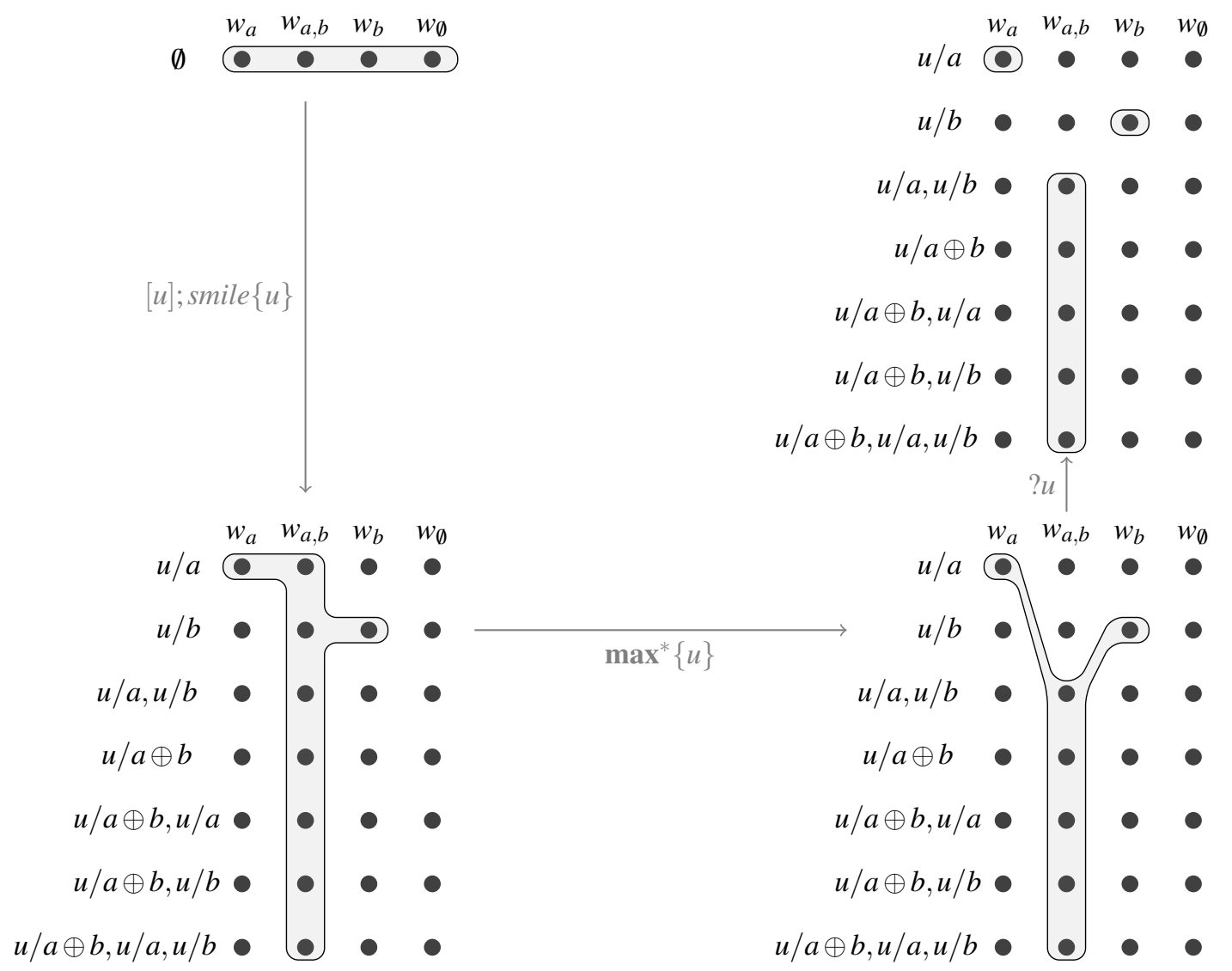

The last example of a single-wh question, given in (30), involves a singular which-phrase, which contributes both $\max ^{*}\{u\}$ and atom $\{u\}$.

$$
\text { Which person smiles? } \leadsto+\left([u] ; \operatorname{atom}\{u\} ; \operatorname{smile}\{u\} ; \max ^{*}\{u\} ; ? u\right)
$$

The update effect of this question is broken up into three steps in the diagram below. First, atom $\{u\}$ ensures that we do not have a plural entity (e.g., $a \oplus b$ ) as the argument of smile. Second, $\max ^{*}\{u\}$ eliminates those possibilities that assign non-maximal plural individuals to $u$. Finally, ? $u$ removes possibilities in which $u$ is assigned a non-unique value. Since all the update functions are in the scope of $\dagger$, the analysis correctly derives that which-phrases trigger a uniqueness presupposition in single-wh questions. Such a uniqueness presupposition is correctly predicted not to be triggered by wh-phrases underspecified for number like who. On our account, a uniqueness presupposition comes about due to the interaction between atom, $\mathbf{m a x}^{*}$, and $? u$ within the scope of $\dagger$. 
A dynamic semantics of single-wh and multiple-wh questions
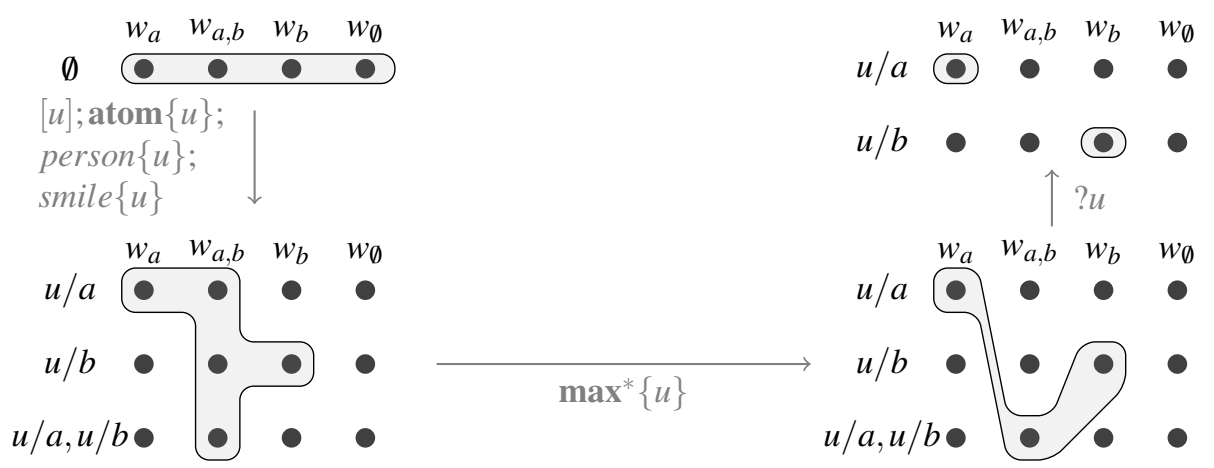

We now turn to a multiple-wh questions with two singular which-phrases.

$$
\begin{aligned}
& \text { Which }{ }^{u_{1}} \text { student is talking to } \text { which }^{u_{2}} \text { professor? } \\
& \leadsto \quad \dagger\left(\left[u_{1}\right] ; \text { student }\left\{u_{1}\right\} ;\left[u_{2}\right] ; \text { professor }\left\{u_{2}\right\} ; \operatorname{atom}\left\{u_{1}\right\} ; \operatorname{atom}\left\{u_{2}\right\} ;\right. \\
& \left.\quad \text { talk_to }\left\{u_{1}, u_{2}\right\} ; \max ^{*}\left\{u_{1}\right\} ; \max ^{*}\left\{u_{2}\right\} ; ? u_{1} u_{2}\right)
\end{aligned}
$$

The update effect of this question is illustrated in the diagram on the next page. The two whichphrases both introduce a discourse referent, $u_{1}$ and $u_{2}$, respectively. The individuals assigned to these drefs by various dref assignment functions are specified on the left side. Sets of assignment functions $G$ belonging to the same possibility are separated from other assignment functions by a horizontal line. Super- and subscripts on a given world specify the extension of the talking-to relation in that world, e.g., $w_{b \sim c}^{a \sim c}$ is a world in which $a$ talks to $c$ and $b$ talks to $c$ and no further talking takes place. For reasons of space we assume that the input context, given on top of the diagram, already contains some information as to who may be talking to whom: it is known that $a$ is talking to $c$, to $d$, or to nobody, and that $b$ is talking to $c$, to $d$, or to both. We furthermore assume that it is known that $a$ and $b$ are students and $c$ and $d$ are professors.

In the diagram, the update is broken down into two steps. The first step leads to a context containing a single alternative, consisting of all possibilities in which $u_{1}$ is a student, $u_{2}$ a professor, $u_{1}$ talks to $u_{2}$ and the values assigned to $u_{1}$ and $u_{2}$ are maximal. The fact that there is only one alternative at this stage means that the context is not yet inquisitive.

The second step is the update contributed by $? u_{1} u_{2}$. This operator raises the issue who talked to whom. Furthermore, ? $u_{1} u_{2}$ eliminates the possibility in the rightmost column. Why is that? Notice that in this possibility, the student assigned to $u_{1}, b$, is talking to several different professors. Thus, there is no functional dependency between the values assigned to $u_{1}$ and those assigned to $u_{2}$ in this possibility. Therefore, ? $u_{1} u_{2}$ removes it. This means that we correctly derive a 'relativized' uniqueness presupposition and avoid the global uniqueness presupposition present in single-wh questions. $^{9}$

9 The account correctly predicts that the 'relativized' uniqueness presupposition disappears in questions with numberneutral wh-phrases like Who is talking to whom?. For reasons of space, we cannot show this in detail but the derivation is similar to that for Who smiles?, in which no uniqueness presupposition arises either. 
$\left[u_{1}\right] ;$ student $\left\{u_{1}\right\} ; \operatorname{atom}\left\{u_{1}\right\}$;

$\left[u_{2}\right] ; \operatorname{professor}\left\{u_{2}\right\} ; \operatorname{atom}\left\{u_{2}\right\}$; talk_to $\left\{u_{1}, u_{2}\right\}$;

$\max ^{*}\left\{u_{1}\right\} ; \boldsymbol{m a x}^{*}\left\{u_{2}\right\}$

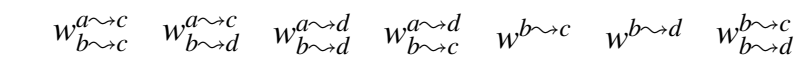

$\emptyset$

○ 000000

$\downarrow$

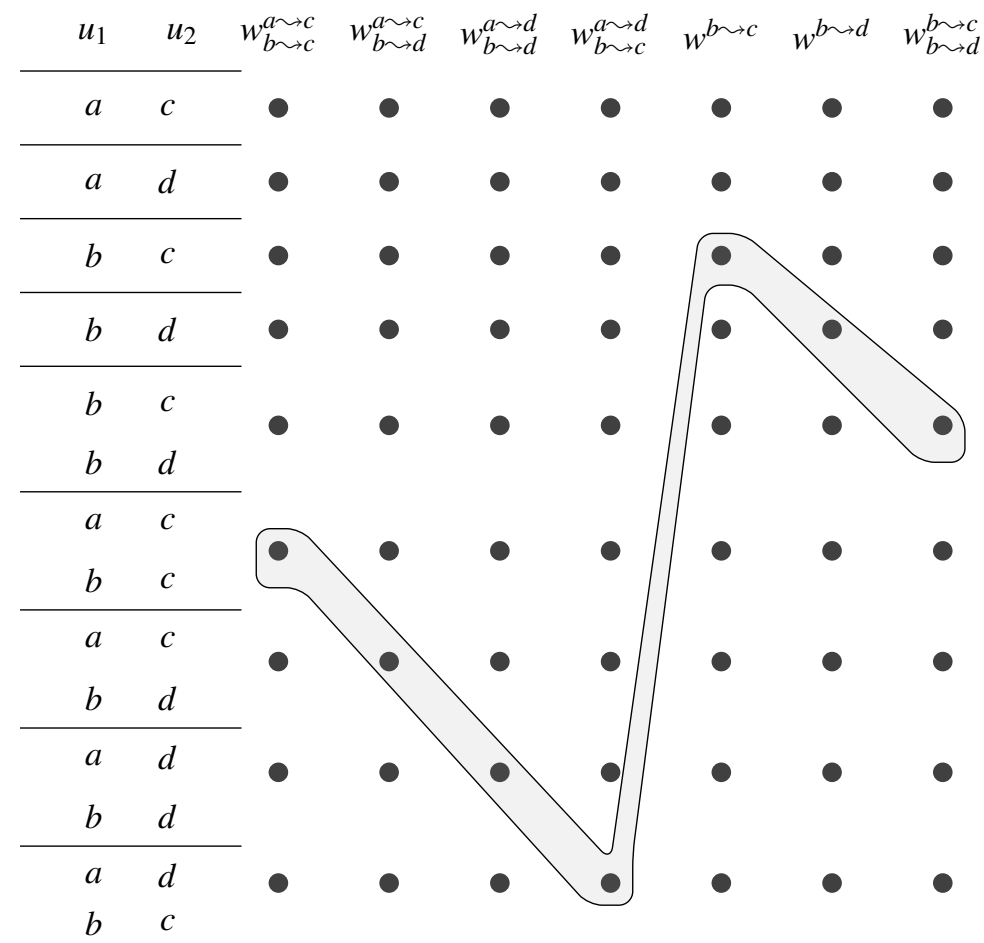

$? u_{1} u_{2}$

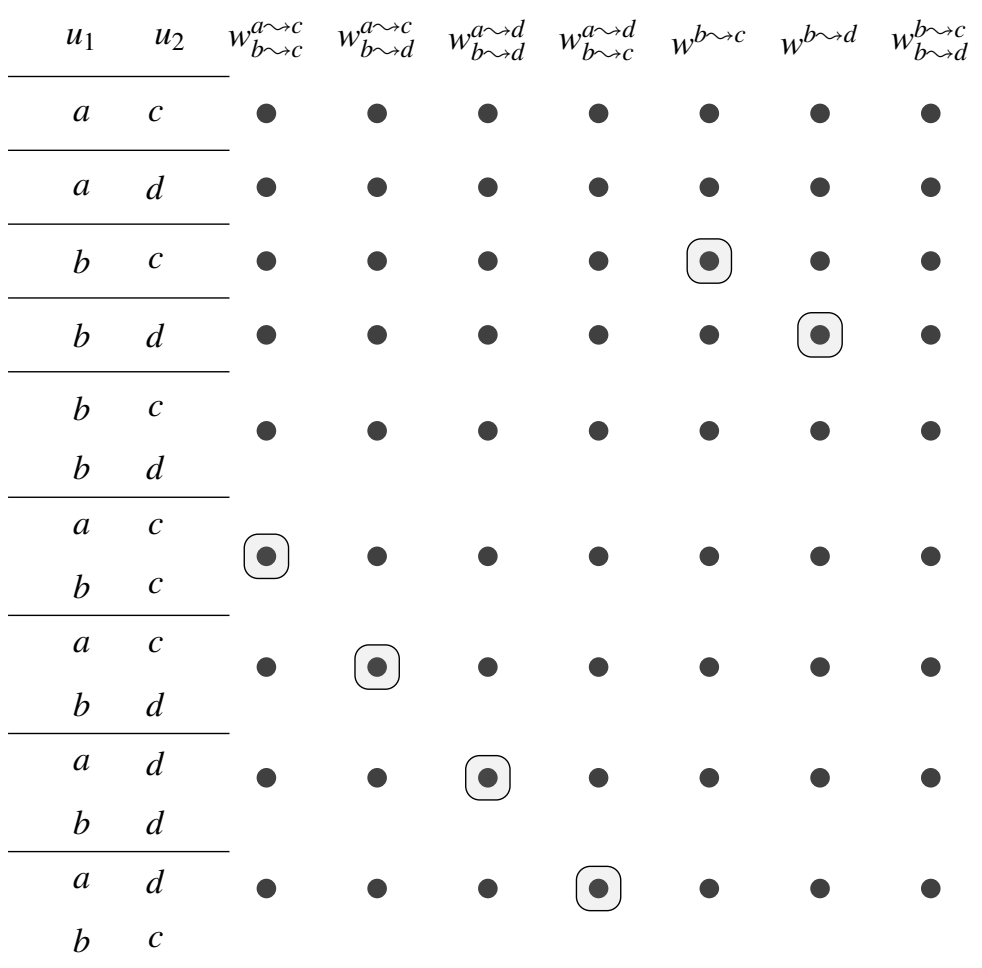


We end with the derivation of the partial mention-some reading of the multiple-wh question in (32). In this example, which herb receives a strong interpretation and where a weak one. As noted above, our account makes it possible to derive this partial mention-some reading because mention-some is not seen as a property of questions as a whole, but rather of individual wh-phrases.

Which herb grows where?

$\leadsto+\left(\left[u_{1}\right] ; \operatorname{herb}\left\{u_{1}\right\} ;\left[u_{2}\right] ; \operatorname{place}\left\{u_{2}\right\} ; \operatorname{atom}\left\{u_{1}\right\} ;\right.$ grow_at $\left.\left\{u_{1}, u_{2}\right\} ; \mathbf{m a x}^{*}\left\{u_{1}\right\} ; ? u_{1} u_{2}\right)$

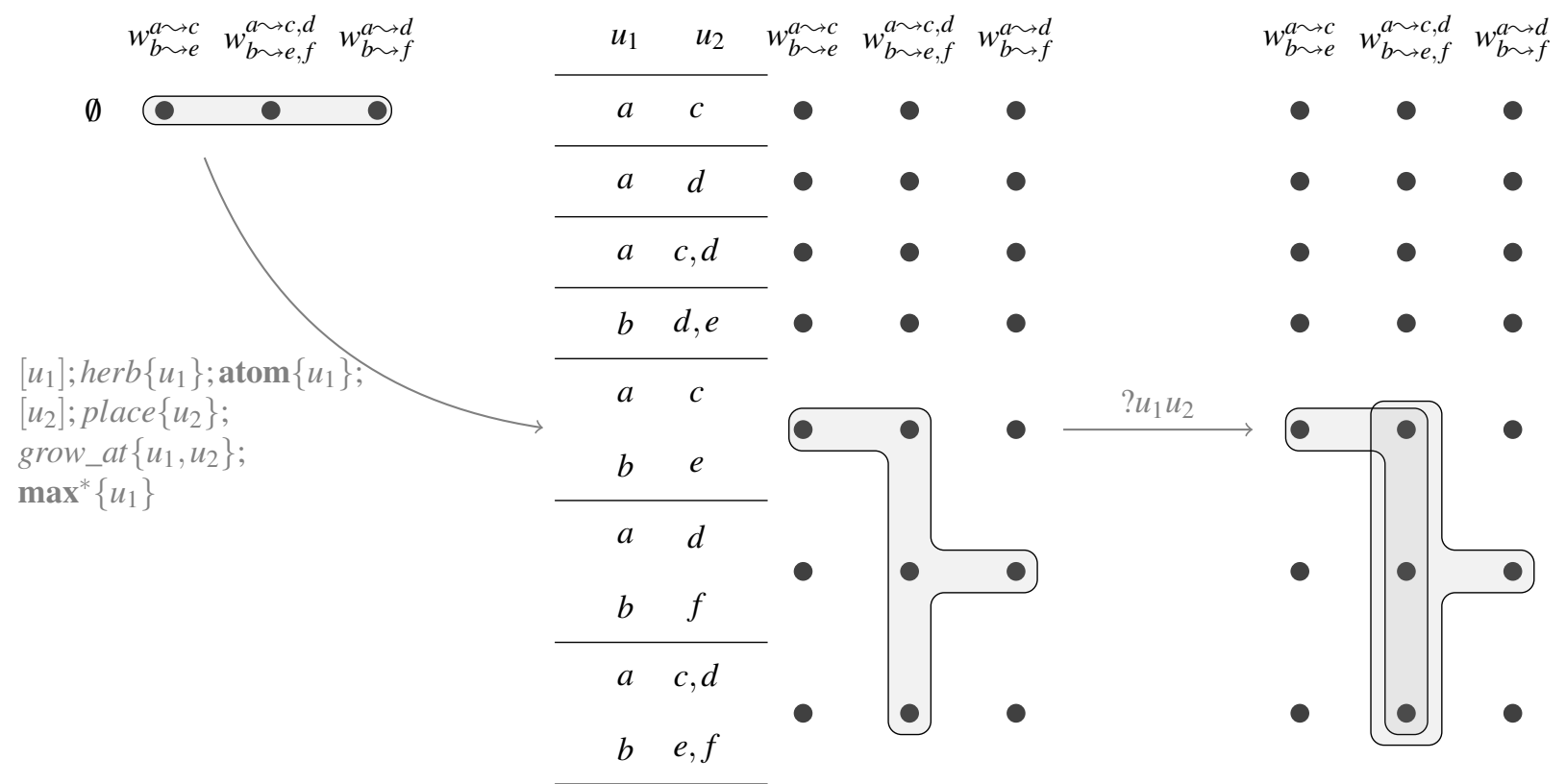

The diagram shows that the partial mention-some interpretation is correctly captured. That is, the derived interpretation is mention-all w.r.t. herbs $\left(u_{1}\right)$ but mention-some w.r.t. locations $\left(u_{2}\right)$. There are two alternatives in the output context: one specifies that herb $a$ grows in location $c$ and herb $b$ grows in location $d$, while the other specifies that $a$ grows in $d$ and $b$ grows in $f$. In both cases, all herbs are covered, but only one location is determined for each of them.

\section{Conclusion and outlook}

We presented an account of single-wh and multiple-wh questions that is uniform, generates mentionsome and mention-all readings in a principled way, and captures effects of number marking. The analysis is couched in dynamic inquisitive semantics. A dynamic framework is useful here because the semantic values it assigns to sentences are more fine-grained than in static proposition-set theories (Hamblin/Karttunen semantics, partition semantics, and static inquisitive semantics). This higher level of fine-grainedness is particularly useful to deal with multiple-wh questions.

This paper is part of a larger effort to develop a dynamic inquisitive semantics framework and explore several of its potential benefits. Further support for a dynamic approach to questions comes from anaphora, intervention effects, and wh-conditionals (Groenendijk 1998; van Rooij 1998; Aloni \& van Rooij 2002; Haida 2007; Dotlačil \& Roelofsen 2019; Li 2019). The recent work of Xiang $(2016,2020 a)$ is in the same spirit, in that it also develops a framework for question semantics which is more fine-grained than proposition-set theories. Her framework is not dynamic, however, but more in the structured meaning tradition (e.g., von Stechow 1991; Krifka 2001). A detailed 
comparison between the two approaches must be left for another occasion, though see Appendix B for some brief remarks in this direction, focusing on multiple-wh questions.

Besides its theoretical contribution, the paper has also made two empirical contributions. First, we observed that multiple-wh questions can have mention-some readings (see also Xiang 2016) but only w.r.t. one of the wh-phrases. We called these partial mention-some readings. Such readings are problematic for many previous accounts of multiple-wh questions. Second, we argued that, while domain exhaustivity is not required in multiple-wh questions (Xiang 2016), domain maximality is. This, too, is a challenge for many previous accounts.

\section{A Formal definitions of extension and subsistence}

Definition 1 (Extending dref assignment functions and matrices).

A dref assignment function $g^{\prime}$ is an extension of another dref assignment function $g$ if and only if $g^{\prime} \supseteq g$. Note that for this to hold it is necessary (but not sufficient) that the domain of $g^{\prime}$ contains the domain of $g$. A dref assignment matrix $G^{\prime}$ is an extension of another dref assignment matrix $G$ if and only if every $g^{\prime} \in G^{\prime}$ is an extension of some $g \in G$ and every $g \in G$ is extended by some $g^{\prime} \in G^{\prime}$. In this case we write $G^{\prime} \geq G$.

Definition 2 (Extending possibilities and information states). A possibility $\left\langle w^{\prime}, G^{\prime}\right\rangle$ is an extension of another possibility $\langle w, G\rangle$ if and only if $w^{\prime}=w$ and $G^{\prime} \geq G$. In this case we write $\left\langle w^{\prime}, G^{\prime}\right\rangle \geq\langle w, G\rangle$. An information state $s^{\prime}$ is an extension of another information state $s$ if and only if every possibility in $s^{\prime}$ is an extension of some possibility in $s$. In this case we write $s^{\prime} \geq s$.

Definition 3 (Subsistence of one information state in another).

If $s, s^{\prime}$ are information states such that $s^{\prime} \geq s$, then we say that $s$ subsists in $s^{\prime}$ if and only if every possibility in $s$ has an extension in $s^{\prime}$.

\section{B Brief comparison with Xiang (2016, 2019, 2020b)}

We cannot pursue a comprehensive comparison here between our proposal and that of Xiang (2016, 2019,2020 b), but we will say a bit more as to why we do not take the latter to provide a uniform account of single-wh and multiple-wh questions. What we mean by this is that the account invokes LF ingredients in the analysis of multiple-wh questions with pair-list readings which are not present in the analysis of single-wh questions. For illustration, consider the following two examples:

(33) Which boy came?

(34) Which boy watched which movie?

The LF that Xiang assumes for (33) is given in (35) and the LF she assumes for (34) under a pair-list reading is given in (36). 

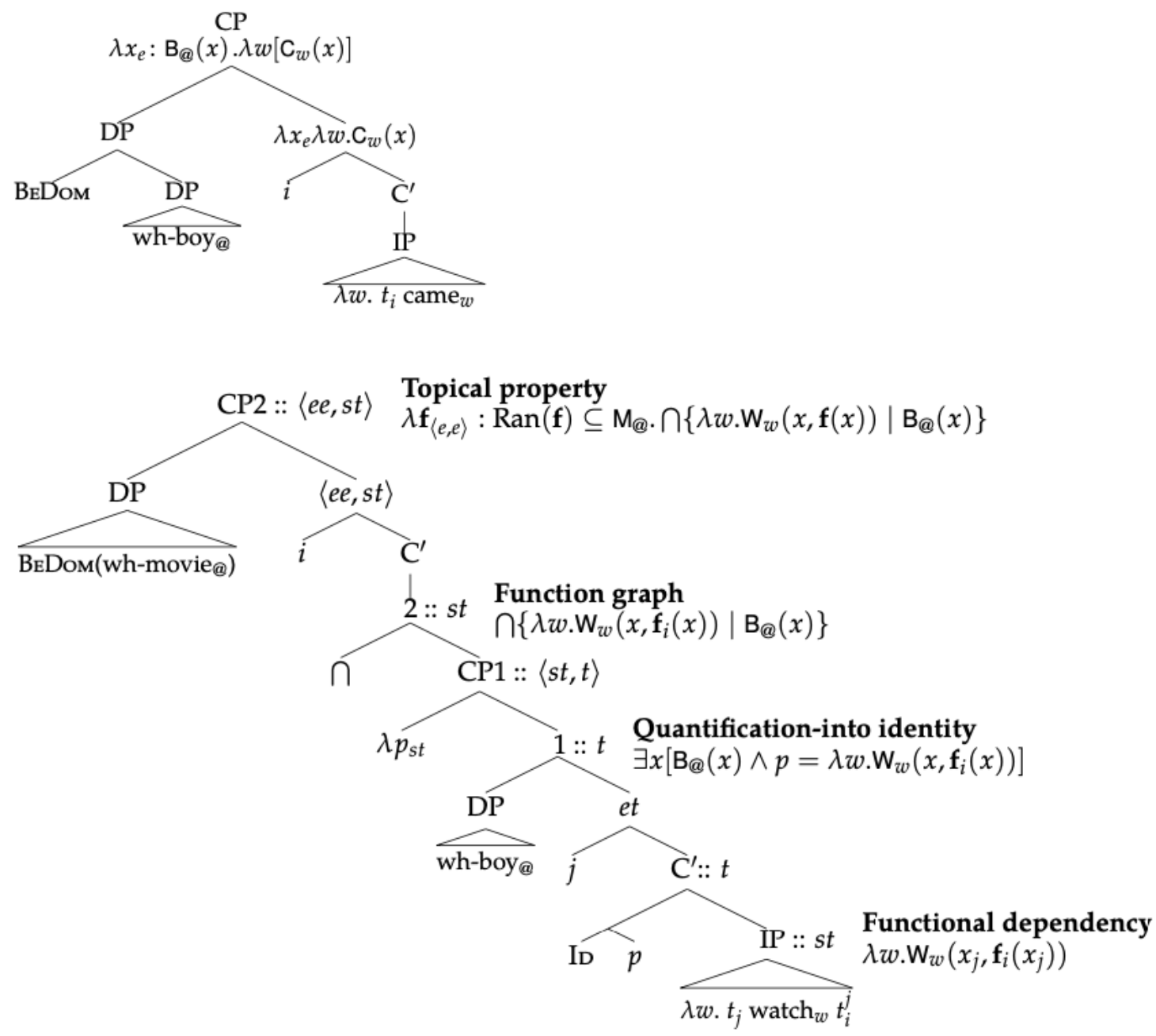

The multiple-wh LF in (36) includes various elements that are not present in the single-wh LF in (35): the $\bigcap$ operator, the ID operator, the node that introduces a free propositional variable $p$ and the one that lambda-abstracts over this variable. There are two further differences between the two LFs as well. First, in (35), the type-shifting operator BEDOM applies to the translation of the wh-phrase which boy, while in (36) this only happens with one of the wh-phrases, which movie, but not with the other, which boy. Second, in (35) the trace of the wh-phrase which boy is treated as a variable of type $e$. In (36) this also holds for the trace of which boy. However, the trace of which movie is treated as a variable of type $(e, e)$. Finally, note that the denotations derived in (35) and (36) differ in semantic type. To derive resolution conditions from these denotations, a polymorphic answer set operator is needed.

On our account all wh-phrases are treated equally, no matter whether they appear in single-wh or in multiple-wh questions, as introducing discourse referents that are bound by a witness request operator. The LFs of single-wh and multiple-wh questions involve exactly the same two left periphery heads, one contributing a witness request operator and the other contributing a presuppositional closure operator. No additional LF operations need to be invoked. 


\section{References}

Aloni, Maria \& Robert van Rooij. 2002. The dynamics of questions and focus. In Brendan Jackson (ed.), Proceedings of salt 12, Cornell University, CLC Publications.

Beck, Sigrid \& Hotze Rullmann. 1999. A flexible approach to exhaustivity in questions. Natural Language Semantics 7(3). 249-298.

van den Berg, Martin. 1996. Some aspects of the internal structure of discourse: the dynamics of nominal anaphora. Amsterdam: University of Amsterdam PhD dissertation.

Brasoveanu, Adrian. 2008. Donkey pluralities: plural information states versus non-atomic individuals. Linguistics and Philosophy 31(2). 129-209.

Champollion, Lucas, Dylan Bumford \& Robert Henderson. 2019. Donkeys under discussion. Semantics and Pragmatics 12.1.

Champollion, Lucas, Ivano Ciardelli \& Floris Roelofsen. 2015. Some questions in typed inquisitive semantics. Talk at workshop on Questions in Logic and Semantics, Amsterdam.

Ciardelli, Ivano, Jeroen Groenendijk \& Floris Roelofsen. 2018. Inquisitive semantics. Oxford University Press.

Dayal, Veneeta. 1996. Locality in Wh-quantification: Questions and Relative Clauses in Hindi. Kluwer Academic Publishers. doi:10.1007/978-94-011-4808-5.

Dayal, Veneeta. 2016. Questions. Oxford University Press.

Dotlačil, Jakub \& Floris Roelofsen. 2019. Dynamic inquisitive semantics: Anaphora and questions. In Proceedings of sinn und bedeutung 23, 365-382.

Fox, Danny. 2012. Multiple wh-questions: uniqueness, pair-list and second order questions. Course notes, MIT.

Fox, Danny. 2013. Mention-some readings of questions. Course notes, MIT.

George, B.R. 2011. Question embedding and the semantics of answers: University of California, Los Angeles PhD dissertation.

Groenendijk, Jeroen. 1998. Questions in update semantics. In Joris Hulstijn \& Anton Nijholt (eds.), Formal semantics and pragmatics of dialogue (Twendial '98), 125-137.

Groenendijk, Jeroen \& Martin Stokhof. 1984. Studies on the semantics of questions and the pragmatics of answers: University of Amsterdam $\mathrm{PhD}$ dissertation.

Groenendijk, Jeroen, Martin Stokhof \& Frank Veltman. 1996. Coreference and modality. In Shalom Lappin (ed.), Handbook of contemporary semantic theory, 179-216. Oxford: Blackwell.

Haida, Andreas. 2007. The indefiniteness and focusing of wh-words. Berlin: Humboldt University $\mathrm{PhD}$ dissertation.

Hirsch, Aron \& Bernhard Schwarz. 2019. Singular which, mention-some, and variable scope uniqueness. In Semantics and linguistic theory, vol. 29, 748-767.

Kratzer, Angelika. 2008. On the plurality of verbs. In Johannes Dölling, Tatjana Heyde-Zybatow \& Martin Schäfer (eds.), Event structures in linguistic form and interpretation, 269-300. Berlin/New York: Mouton de Gruyter.

Krifka, Manfred. 1989. Nominal reference, temporal constitution, and quantification in event semantics. In Renate Bartsch, Johan van Bentham \& Peter van Emde Boas (eds.), Semantics and contextual expressions, Dordrecht: Foris.

Krifka, Manfred. 2001. For a structured meaning account of questions and answers. Audiatur Vox Sapientia. A Festschrift for Arnim von Stechow 287-319.

Landman, Fred. 2000. Events and Plurality: The Jerusalem Lectures. Dordrecht: Kluwer. 
Li, Haoze. 2019. Deriving short answers from Hamblin sets: A case study of Mandarin whconditionals. In Semantics and linguistic theory, vol. 29, 138-159.

Muskens, Reinhard. 1996. Combining Montague semantics and discourse representation. Linguistics and Philosophy 19(2). 143-186.

Rizzi, Luigi. 1997. The fine structure of the left periphery. In L. Haegeman (ed.), Elements of grammar, 281-337. Dordrecht: Kluwer.

Rizzi, Luigi. 2001. On the position int(errogative) in the left periphery of the clause. In Current studies in Italian syntax: Essays offered to Lorenzo Renzi, 287-296.

Roelofsen, Floris. 2015. The semantics of declarative and interrogative lists. Manuscript, ILLC, University of Amsterdam.

van Rooij, Robert. 1998. Modal subordination in questions. In Proceedings of twendial, 237-248.

von Stechow, Arnim. 1991. Focusing and backgrounding operators. In Werner Abraham (ed.), Discourse particles, 37-84. Amsterdam: John Benjamins.

Theiler, Nadine. 2014. A multitude of answers: Embedded questions in typed inquisitive semantics. University of Amsterdam, MSc thesis.

Xiang, Yimei. 2016. Interpreting questions with non-exhaustive answers: Harvard University PhD dissertation.

Xiang, Yimei. 2019. Getting quantifying-into questions uniformly: Functionality, domain exhaustivity, and quantificational variability. In Semantics and linguistic theory, vol. 29, 160-179.

Xiang, Yimei. 2020a. A hybrid categorial approach to question composition. Linguistics and Philosophy.

Xiang, Yimei. 2020b. Quantifying into wh-dependencies: Composing multi-wh questions and questions with quantifiers. Manuscript, Rutgers University, March 2020.

Zhao, Zhuoye. 2019. Varieties of distributivity: From Mandarin dou to plurality, free choice and scalarity. MSc Thesis, University of Amsterdam.

Jakub Dotlačil

Trans 10

3512JK Utrecht

Netherlands

j.dotlacil@gmail.com
Floris Roelofsen

ILLC, University of Amsterdam

Postbus 94242

1090 GE Amsterdam

Netherlands

f.roelofsen@uva.nl 